American Journal of Pediatrics
2020; 6(2): $91-93$
http://www.sciencepublishinggroup.com/j/ajp
doi: $10.11648 /$ j.ajp.20200602.14
ISSN: 2472-0887 (Print); ISSN: $2472-0909$ (Online)

Case Report

\title{
Neurocysticercosis in a Child Living in the Urban Community of Yaoundé, Cameroon: A Case Report in a Low Resource Setting
}

\author{
Georges Pius Kamsu Moyo*, Audrey Thérese Mbang, Laura Kuate Makowa, \\ Raïssa Monayong Mendomo, Sonia Zebaze, Hubert Désiré Mbassi Awa \\ Department of Paediatrics, Faculty of Medicine and Biomedical Sciences, University of Yaoundé I, Cameroon
}

\author{
Email address: \\ kamsuzicfried@yahoo.fr (G. P. K. Moyo) \\ ${ }^{*}$ Corresponding author
}

\section{To cite this article:}

Georges Pius Kamsu Moyo, Audrey Thérese Mbang, Laura Kuate Makowa, Raïssa Monayong Mendomo, Sonia Zebaze, Hubert Désiré Mbassi Awa. Neurocysticercosis in a Child Living in the Urban Community of Yaoundé, Cameroon: A Case Report in a Low Resource Setting. American Journal of Pediatrics. Vol. 6, No. 2, 2020, pp. 91-93. doi: 10.11648/j.ajp.20200602.14

Received: February 7, 2020; Accepted: February 20, 2020; Published: March 6, 2020

\begin{abstract}
Background: Neurocysticercosis is a helminthiasis of public health interest in developing countries, where it is potentially responsible for $70 \%$ of epilepsy cases. Clinical presentations are diverse and depend on central nervous system localization of the parasite. The diagnosis is based on a number of factors including environmental context, clinical presentation, radiological imaging and serology. The treatment is often medical, with surgery being left for specific cases. Holistic prevention involves prophylaxis, treatment of asymptomatic carriers and reinforcement of health education. Method: We report a case of neurocysticercosis being responsible for a curable form of epilepsy in a Cameroonian child living in the urban community of Yaoundé. The patient was treated exclusively by medical means involving curative and symptomatic medications over a period of 21 days. Results: By the end of the treatment, the patient clinically recovered, though some residual latent cerebral sequels persisted. Conclusion: Neurocysticercosis may be found in children living in urban communities, causing neuropsychic disorders among which epilepsy. Prompt diagnosis may be aided by cerebral radiological imaging such as CT-scan or MRI. The management may be exclusively medical with complete recovery. However, primary prevention is a relevant intervention that may be done by proper disposal of human and animal faeces, rigorous hygiene, effective meat cooking before consumption, health education and prophylaxis with anthelmintics.
\end{abstract}

Keywords: Cysticercosis, Neurocysticercosis, Epilepsy, Cameroon

\section{Background}

Neurocysticercosis is a disease caused by the encystment of cysticercus larvae in the central nervous system tissues [1]. The number of infected persons is estimated between 20 and 50 million worldwide, occurring mainly in rural areas [1]. The sickness could remain asymptomatic in $20 \%$ of cases [2]. Some common manifestations are convulsion, headache, vomiting, psychic [2].

\section{Case Report}

This is the case of a 4 years old male child who presented with frequent generalized afebrile convulsive seizures over a period of three months. The child's relevant history revealed he was never given prophylaxis against worm infection. There was a notion of frequent consumption of poorly cooked pork meat. The existence of a pigsty less than $5 \mathrm{~m}$ away from family house where the child used to play. The child had slightly altered developmental milestones, was a nursery school pupil whose teachers complained of poor writing and reading skills, unusual restlessness and aggressiveness. On physical examination The child was agitated, hyperactive with difficulties to stay focus. The initial work-up consisted of an electroencephalogram, blood electrolytes, glycaemia and full blood count which were all normal. The cerebral C.T-scan showed association of micro 
calcifications dispersed throughout the brain with cysts and annular contrast representing surrounding edema. These features were in favor of neurocysticercosis at different developmental stages. Based on C.T-scan results, cysticercosis serology was asked and stool exam equally done in search of adult worm or other forms of parasite. The final serology exams were all positive for cysticercosis, no adult worm, larvae or egg were found in stool. We thus concluded as neurocysticercosis.

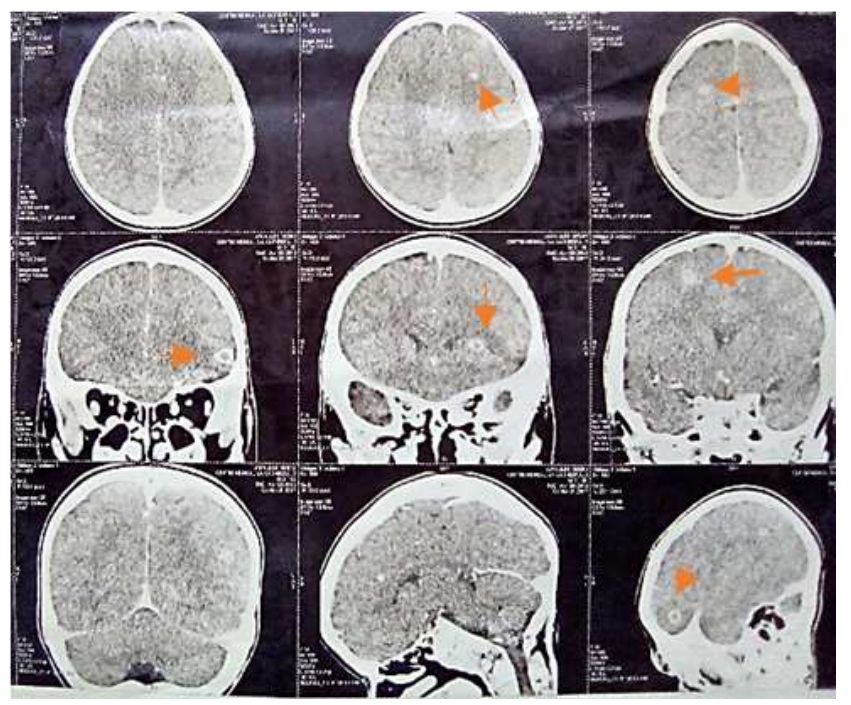

Figure 1. Cerebral lesions before treatment.

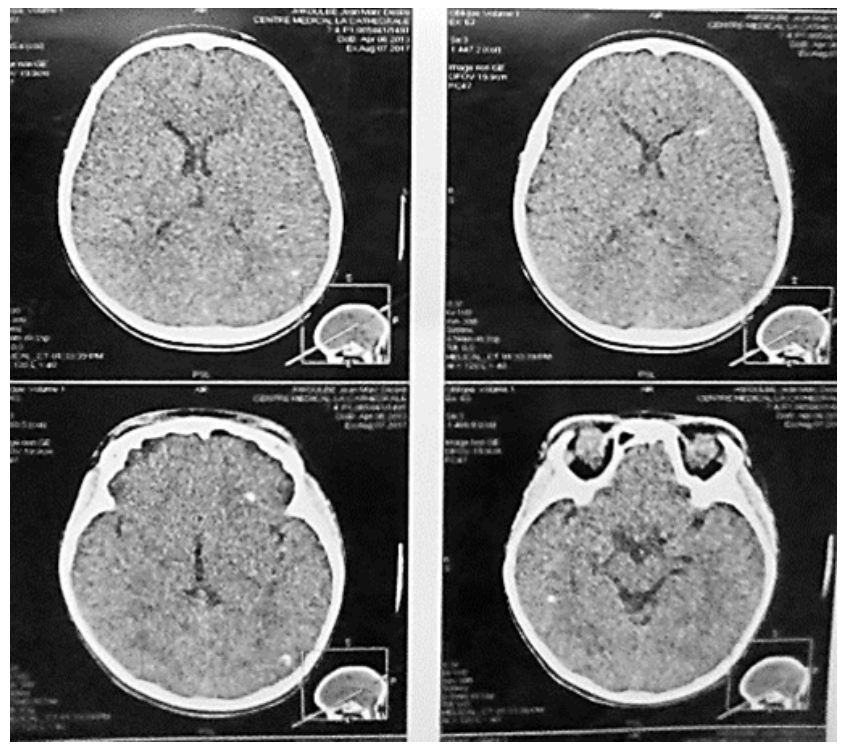

Figure 2. Brain after treatment.

The child was initially put on sodium valproate protocol at $30 \mathrm{mg} / \mathrm{kg}$ but was replaced five days later with carbamazepine at $15 \mathrm{mg} / \mathrm{kg} /$ day after the child had developed a rash. Prednisone was prescribed at $1 \mathrm{mg} / \mathrm{kg} /$ day for fifteen days with calcium at $250 \mathrm{mg} /$ day, aluminum and magnesium hydroxides combined at $10 \mathrm{ml}$ thrice daily. Albendazol was prescribed for 21 days at $15 \mathrm{mg} / \mathrm{kg} /$ day. The child was reassessed at the end of the treatment with considerable amelioration, no more seizures, considerable improvement on child's behavior and school performances was noted. Blood electrolytes, glycaemia and full blood count controls were normal. The cerebral C.T scan control showed persistent calcifications throughout the brain parenchyma, but marked disappearance of cysts and edema. Carbamazepine was progressively stopped without reappearance of seizures.

\section{Discussion}

Neurocysticercosis is a clinical form of cysticercosis disease caused by the encystment of cysticercus larvae in the central nervous system tissues [1]. It is presently responsible for 50000 deaths every year worldwide [1]. One of the world's greatest prevalence is in Madagascar with about $10 \%$ of the population being contaminated [3]. Neurocysticercosis is popularly believed to be a disease of the poor and rural areas, persisting mainly where there is human proximity with pigs and poor disposal of feces $[1,4]$ Other risk factors are low level of health education, poor hygiene conditions [4]. Its prevalence is thus high in Africa, southern America and Asia [1]. These conditions best describe the environmental context of our patient who had several risk factors involving the neighbor's pigsty where the child used to play, frequent consumption of insufficiently cooked pork, and the patient's age which shows his level of education and the difficulty to observe good hygiene. Neurocysticercosis affects individuals of both sexes, with an average age of diagnosis between 30 and 40 years old [1]. It is rare before 2 years of age and after 60 years [1]. The patient was 4 years old at the time of the diagnosis suggesting recent contamination. This may explain why no evidence of adult worm presence was found in stool exam. The sickness may remain latent in $20 \%$ of cases, making the overall prevalence an underestimation and the necessity for treatment of carriers for prophylaxis $[2,5]$. The most common symptom is convulsion which may become recurrent leading to epilepsy [1]. Neurocysticercosis is responsible for close to $56 \%$ of epilepsy cases in Cameroon [6]. This was the case of our patient who suffered repeated generalized seizures over three months. Symptoms could be diverse according to the localization of the parasite in the central nervous system [7] or the importance of the surrounding edema. Psychic manifestations and developmental milestones delay are also common in children [8]. In this case, the child presented behavioral imbalances such as restlessness, agitation, hyperactivity and equally difficulties reading and writing.

Paraclinical exams have a major role in the management of neurocysticercosis [2]. They are important for the diagnosis and give clues for the prognosis of the sickness [9]. The most contributive work-ups are electroencephalogram (E. E. G), magnetic resonance imaging (MRI), CT-scan and blood serology [1]. Cerebrospinal fluid (C. S. F) analysis may equally be important [1] and E. E. G is pertinent for identifying epileptic trigger zones [10]. In the present case E. E. G was normal as in $60 \%$ of cases $[1,10]$. This could be explained by the fact that the child wasn't convulsing for the 
first time so E. E. G sensitivity and specificity could not be totally reliable [10]. Radiological imaging, mainly MRI and cerebral CT-scan may be of great importance for the diagnosis of neurocyticercosis [11]. Because of financial difficulties, CT-scan was more affordable in our context. Radiological imaging shows the topographical diagnosis, permit appreciation of the severity of the lesions and enables to rule out differentials [11]. Typical lesions are those of intracerebral calcifications, cysts and surrounding edema $[11$, 12]. This corresponds to simple active stage of neurocysticercosis according to Aguilar-Rebolledo radiological classification [13]. CT- scan has the additional advantage to show calcifications [11], but MRI is best to identify cysts, scolex, C. S. F and meninges involvement, ophthalmological and muscle cysts [14]. Cysticercosis serology by ELISA was positive, and further comforted the diagnosis of neurocysticercosis [1]. Lumber puncture wasn't performed, and so C. S. F and meninges involvement could not be completely excluded.

Concerning the treatment of such simple active neurocysticercosis, both symptomatic and etiological treatments are recommended $[9,13]$. Appropriate monitoring and management of drug side effects are also to be considered. The pathophysiology here is related to toxic and immuno-allergic effects due to the parasite, inflammation generating edema and mechanical effects [1]. Concerning our patient, the symptomatic treatment comprised carbamazepine as antiepileptic. The curative treatments consisted of prednisone to reduce cerebral edema, as an anti-inflammatory drug with additional anti-allergic actions [9]. Calcium supplementation and gastric protection were given to prevent corticosteroid side effects. Albendazol was preferred over praziquantel as anthelmintic because of its availability, safety and efficacy. Moreover, it could have synergic effects when associated with corticosteroids and antiepileptic drugs [1, 9, 15]. Surgical intervention mostly meant for particular cases of voluminous cysts or hydrocephalus was of no indication in this case [1].

\section{Conclusion}

Neurocysticercosis is a helminthiasis of public health interest in developing countries, where it is potentially responsible for $70 \%$ of epilepsy cases. Clinical presentations are diverse and depend on central nervous system localization of the parasite. The diagnosis is based on a number of factors including environmental context, clinical presentation, radiological imaging and serology. The treatment is often medical, with surgery being left for specific cases. Holistic prevention should involve prophylaxis, treatment of asymptomatic carriers and reinforcement of health education.

\section{Funding}

Private.

\section{Conflict of Interest}

None.

\section{Acknowledgements}

Hospitals authorities, all collaborators to this project.

\section{References}

[1] Lagueste M: La neurocysticercose humaine en 2015: etude retrospective de 4 cas pris en charge au CHU de Rouen de 2011 à 2015. Thesis. Université de Rouen 2015; 122p.

[2] Singhi P, Singhi S. Neurocysticercosis in children. J Child Neurol, 2004; 19: 92-482.

[3] Scott J X, Devia A et al. A rare presentation of neurocysticercosis in a young child. J Trop Pediatr, 2005; 51: $5-254$.

[4] Castillo H. imaging of neurocysticercosis. Semin Roentgenol, 2004; 39: 73-465.

[5] Del Brutto O H, Garcia H H. Neurocysticercosis in non endemic countries: time for a reappraisal. Neuroepidemioogy, 2012; 39: 145-6.

[6] Zoli A D, Nguemkam et al. Neurocysticercosis and epilepsy in Cameroon. Trans R Soc Trop Med Hyg, 2003; 97 (6): 683-6.

[7] Coyle C, Wittner $M$ et al. Tropical infectious diseases: principles, pathogens and practice. Philadephia: Churchill Livingstone Guerant R, Walker D Weller P, editors 1999.

[8] Ramirez-Bernudez $\mathbf{J}$, Higuera $\mathrm{J}$ et al. Is dementia reversible in patients with neurocysticercosis. J Neurol Psychiatr, 2005; 76: 1164-6.

[9] Neurocysticercosis in sub-saharan Africa: a review of prevalence, clinical characteristics, diagnosis and management. Technical University of munich, Bavaria, Germany.

[10] Diagana M, Nsengiyumva G. Electroencephalogrammes réalisées chez 250 patients dans une zone d'endemie cysticerquienne au Burundi. Neurophysiol Clin, 2005; 35: $1-10$.

[11] Zhao S, Xu K. Changes in computed tomogram in cerebral parenchyma cysticercosis treated with albendazole. Zhon Ch Xue Jsh Ch Bi Z Zhi 1999; 390-3.

[12] Talukdar B, Saxena A et al. neurocysticercosis in children: clinical characteristics and outcome. Am Trop Pediatr 2002; 22 333-9.

[13] Aguilar-Rebollero F, Meza-Lucas A et al. Evaluation of enzyme linked immunoelectrotransfer blot essay for diagnostic of neurocysticercosis in children. J Child Neurol, 2002; 17: 20-4016.

[14] Agrawal S, Ranjam S et al. Ocular myocysticercosis: an unusual case of ptosis. Nepal J Ophtalmo, 2013; 5: 81-279.

[15] Garcia H H, Gonzales I et al. Efficacy of combined antiparasitic therapy with praziquantel and albendazol for neurocysticercosis: a double-blind randomized controlled trial. Lancet Infect Dis, 2014; 14: 687-95. 